\title{
Developing a concise set of principles for use by clinicians and regulators to determine the need for treatment within a compensable setting
}

Paul Coburn

Victorian WorkCover Authority \& Transport Accident Commission, Melbourne VIC 3000, Australia. paul_coburn@vwa.vic.gov.au

Background: Delivering quality health services in a cost effective manner is becoming an increasingly critical issue for insurance companies and government agencies. The Clinical Framework used as part of peer review has been proposed as an alternative for institutions to control costs and maintain optimal clinical care.

Objectives: To develop a concise set of principles for use by clinicians and regulators to determine the need for treatment within in a compensable setting.

Method: The Clinical Framework was first implemented in 2003 at the Victorian WorkCover Authority as part of peer review of physiotherapy management of injured workers. It consists of five principles that clinicians and institutions use to determine the ongoing need for intervention. The Framework allows clinicians to exercise judgment in selecting treatment with due consideration to the evidence and patient's individual needs. Results: Following its introduction in 2003, it broadened to apply to chiropractic, osteopathy, psychology, and occupational therapy. In 2012 it was endorsed across Australia for motor accident and worker's compensation bodies by the representative bodies of the above disciplines. In 2013 Federal Minister Shorten released a review of the SRC Act which recommended amendments including "medical treatment must meet objective standards such as those in the Clinical Framework."

Discussion: This paper will discuss some of the issues that have been addressed in implementing the Clinical Framework on over 20,000 files over the past ten years.

Conclusions: The Clinical Framework is a document that has won broad acceptance across the Australian compensable system in allied health as part of sustaining quality care while reducing unnecessary costs. 